\title{
Preoperative short-term fasting protects liver injury in patients undergoing hepatectomy
}

\author{
Chuanfei Zhan", Xinzheng Dai", Gefengqiang Shen, Xu Lu, Xuehao Wang, Ling Lu, Xiaofeng Qian, \\ Jianhua Rao
}

Hepatobiliary/Liver Transplantation Center, The First Affiliated Hospital, Nanjing Medical University, Nanjing 210029, China

Contributions: (I) Conception and design: J Rao, X Qian, C Zhan; (II) Administrative support: X Wang; (III) Provision of study materials or patients: L Lu; (IV) Collection and assembly of data: X Dai, G Shen; (V) Data analysis and interpretation: X Lu; (VI) Manuscript writing: All authors; (VII) Final approval of manuscript: All authors.

\#These authors contributed equally to this work.

Correspondence to: Jianhua Rao; Xiaofeng Qian. Hepatobiliary/Liver Transplantation Center, The First Affiliated Hospital of Nanjing Medical University, Key Laboratory on Living Donor Liver Transplantation of National Health and Family Planning Commission, Guangzhou road \#300, Nanjing 210029, China. Email: raojh@njmu.edu.cn; qianxf@163.com.

Background: Our previous study demonstrated that preoperative short-term fasting attenuates mice hepatic ischemia/reperfusion injury (IRI), which greatly piqued our interest in verifying if fasting produces similar protective effects in patients undergoing hepatectomy.

Methods: Eighty patients with liver tumors were randomized into control (Ctrl, $n=40$, preoperative fasting for $6 \mathrm{~h}$ ) or fasting group (Fasting, $\mathrm{n}=40$, preoperative fasting for $24 \mathrm{~h}$ ). Serum was collected at pre-operation (Pre-Op), post-operation 1 day (POD-1), post-operation 3 days (POD-3), and post-operation 7 days (POD-7). Liver tissue was removed from the resected specimen.

Results: Sixty-three patients were eventually enrolled, with 33 in Ctrl and 30 in Fasting group. Our data showed that $24 \mathrm{~h}$ fasting effectively attenuated elevated sALT and sAST levels after operation $(\mathrm{P}<0.05)$, but serum total bilirubin was significantly lower at only POD-3 $(\mathrm{P}<0.05)$; and serum albumin was not markedly different in either of the groups. Interestingly, $24 \mathrm{~h}$ fasting partially attenuates expression of pro-inflammatory cytokine (TNF- $\alpha$ ) and improves oxidative stress (MDA and SOD). Our data further showed short-term fasting triggered Nrf2 signaling pathway.

Conclusions: This study demonstrates preoperative short-term fasting effectively improves clinical outcomes and markedly attenuates inflammatory responses and oxidative stress in patients undergoing hepatectomy, and Nrf2 signaling pathway may play a key role in fasting against inflammatory responses and oxidant stress.

Keywords: Short-term fasting; hepatectomy; inflammatory cytokines; oxidative stress; nuclear factor erythroid-derived 2-related factor 2 signaling ( $\mathrm{Nrf} 2$ signaling)

Submitted Jun 05, 2018. Accepted for publication Oct 16, 2018.

doi: 10.21037/atm.2018.10.64

View this article at: http://dx.doi.org/10.21037/atm.2018.10.64

\section{Introduction}

The temporary occlusion of the hepatic inflow (pringle maneuver) or the hepatic inflow and outflow (total hepatic vascular occlusion) is a common method used during liver resection or liver transplantation. The prolonged interruption results in liver ischemia/reperfusion injury
(IRI) caused by progressive hepatocellular injury and death after reperfusion, which is considered to be a risk factor for recent and distant damage to residual liver or transplant liver (1-3). The development of a protective strategy against IRI is warranted to alleviate the consequences of hepatic IRI. Calorie restriction (CR) and fasting have 


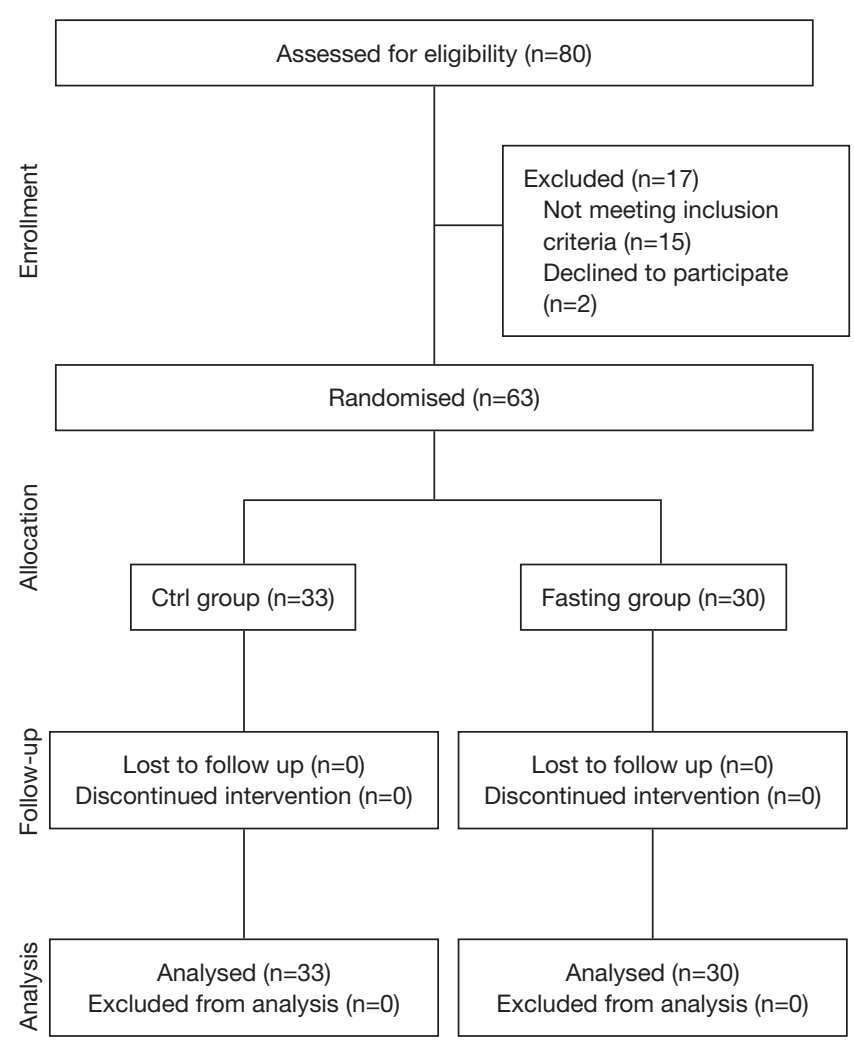

Figure 1 Flowchart of patient enrollment, allocation follow-up and analysis.

displayed some beneficial effects on the prolongation of life and increased resistance to stress. In rodents, CR increases resistance to paraquat toxicity (4). Further, CR is also associated with improved resistance against multiple stressors, such as IRI to the kidney, liver, brain, and heart (5-7). Dietary restriction (DR), which is defined as reduced food intake without malnutrition, is a commonly used CR method, but it is not easily implemented in the clinical setting due to the length of time required (8-11). Interestingly, fasting can rapidly produce similar benefits to long-term DR in terms of gene expression, physiology, and stress resistance. It has recently been reported that fasting can rapidly induce DR-like effects on hepatic IRI. In fact, fasting for 3 days has been shown to be as effective as a month of DR in reducing IRI, which implies a new and promising noninvasive strategy for protecting the liver against the detrimental effects of hepatic IRI (12). Our previous study demonstrated that short-term starvation effectively attenuated liver IRI by inhibiting hepatocellular apoptosis in the mouse liver IRI model (1). In the present study, we aimed to verify whether preoperative short-term fasting could induce similar protective effects in patients undergoing hepatectomy.

\section{Methods}

\section{Subjects and clinical design}

This is a prospective, single-blinded, randomized study. The Research Ethics Committee of the First Affiliated Hospital of Nanjing Medical University, in Nanjing, China (Ref: 2014/185) granted permission for the study protocol, and the trial was registered at the beginning of the study (Ref: ChiCTR-TRC-11001530, September 2014). The research was performed from November 2015 to May 2016 in the First Affiliated Hospital of Nanjing Medical University. Patients with liver tumors were enrolled in this study when they were scheduled for a hepatectomy with inflow occlusion and informed consent was signed by each participant. We used the following inclusion criteria: (I) Child-Pugh classification for A, and (II) hepatectomy with Pringle maneuver, and the exclusion criteria: (i) $<18$ and $>75$ years old; having any of the following conditions: being completely blind and deaf; unconsciousness; Alzheimer's disease; anorexia nervosa; alcoholic insanity; epilepsy; schizophrenia; paralytic disease; serious chronic infection; extensive metastasis tumor; AIDS; gastric ulcer with hemorrhage; pregnancy and breastfeeding; weakness; malnutrition; diabetes type 1 ; diabetes type 2 requiring continuous insulin injections; cardiac failure; arrhythmia; uremia; or secondary hypertension (13-16); and (ii) intraoperative additional implementation of hepatic artery ligation. All patients were randomly divided into the control group (Ctrl group, traditional fasting) or the fasting group (Fasting group, preoperative fasting for $24 \mathrm{~h}$ ). The randomization sequence was computer generated, and patients were distributed in a table of simple random numbers, which was showed in a CONSORT Figure (Figure 1).

\section{Surgery}

All of the operations were conducted by the same medical team to minimize bias. The operation was classified into 2 grades based on the extent of liver resection: Grade I was defined as local resection of the tumor, while Grade II was defined as a combined liver segmentectomy, including hemihepatectomy. After mobilization of the liver, inflow occlusion was performed with a 4-mm Mersilene 
Table 1 Comparison of the demographic data between the Fasting group and the Ctrl group

\begin{tabular}{|c|c|c|c|}
\hline Characteristic & Fasting group $(\mathrm{n}=30)$ & Ctrl group $(n=33)$ & $P$ value \\
\hline Maximum tumor diameter (cm) & $6.12 \pm 3.19$ & $6.18 \pm 3.16$ & 0.93 \\
\hline Multiple tumors N/P (n) & $24 / 6$ & $28 / 5$ & 0.61 \\
\hline BCLC stage $A / B$ & $27 / 3$ & $30 / 3$ & 0.90 \\
\hline Platelets (104/aL) & $10.53 \pm 4.17$ & $10.15 \pm 3.89$ & 0.39 \\
\hline Prothrombin time (\%) & $79.40 \pm 11.59$ & $76.39 \pm 10.28$ & 0.28 \\
\hline AFP (ng/dL) & $187.30 \pm 286.83$ & $284.36 \pm 330.71$ & 0.22 \\
\hline ICG-15, \% & $6.97 \pm 1.92$ & $4.39 \pm 2.00$ & 0.25 \\
\hline Total operation time (min) & $100.83 \pm 13.00$ & $103.43 \pm 14.48$ & 0.42 \\
\hline Blood loss (mL) & $157.50 \pm 87.56$ & $144.24 \pm 94.23$ & 0.56 \\
\hline IBT N/P (n) & $29 / 1$ & $32 / 1$ & 0.95 \\
\hline Grade I/II resection (n) & $23 / 7$ & $28 / 5$ & 0.4 \\
\hline
\end{tabular}

BCLC, Barcelona-CI Liver Cancer; N/P, negative/positive; IBT, intraoperative blood transfusion; JIS, The Japan Integrate Staging.

tape around the hepatic pedicle. Time of continuous inflow occlusion was determined according to the required operation. We excluded patients with additional intraoperative implementation of hepatic artery ligation and other peculiar vascular clamping manners, which are considered to have great influence on postoperative outcomes (17). All of the complications occurring during and after surgery were recorded, such as blood loss, bile leaks, infections, and so on. All of the patients received similar treatment after surgery in the same medical unit, and were followed for their entire hospitalization.

\section{General data and hepatic function}

General data of patients and intraoperative and postoperative complications were collected in an electronic medical record system (SimLink Information System Co., Ltd., China) as shown in the study flow chart (Table 1, Figure 1). The elevation of serum aminotransferase occurs when liver cells are subjected to acute damage; hepatic IRI is an acute injury, so we chose hepatic function to reflect the real extent of IRI directly (18). Hepatic function was determined before and after the operations by Automatic Biochemistry Analyzer (Beckman Kurt Co., Ltd., USA).

\section{Enzyme-linked immunosorbent assay (ELISA)}

Serum TNF- $\alpha$, IL-6, and IL-10 secretions were measured by ELISA kits (eBioscience Affymetrix Inc., USA) according to the manufacturer's standard protocols, which principally involved the mechanism of inflammation of hepatic IRI. Absorbance was read on a Multiscan FC plate reader and analyzed with SkanIt for Multiscan FC software (Thermo Scientific, Schwerte, Germany).

\section{Quantitative real-time PCR}

Liver tissue was harvested from the tissue adjacent to the tumor. Total RNA was extracted from liver tissue samples that had been frozen at $-80^{\circ} \mathrm{C}$ using a TRIzol kit following the manufacturer's protocol (Invitrogen, Shanghai, China), and RNA concentration was measured by a spectrophotometer (Thermo Scientific, Schwerte, Germany). cDNA was synthesized according to the manufacturer's instructions using HiScriptII Q RT SuperMix (+gDNA wiper) (Vazyme, USA). Polymerase chain reaction (PCR) was performed with SYBR Green Master Mix (High ROX Premixed) (Vazyme, USA). using the following primers: TNF- $\alpha$ (5'-CCT CTC TCT AAT 
Table 2 Comparison of the postoperative complications between the Fasting group and the Ctrl group

\begin{tabular}{lccc}
\hline Characteristic & $\begin{array}{c}\text { Fasting group } \\
(\mathrm{n}=30)\end{array}$ & $\begin{array}{c}\text { Ctrl group } \\
(\mathrm{n}=33)\end{array}$ & P value \\
\hline Hemorrhage $(\mathrm{n})$ & 0 & 1 & 0.34 \\
Biliary leakage $(\mathrm{n})$ & 1 & 0 & 0.30 \\
Ascites $(\mathrm{n})$ & 2 & 3 & 0.72 \\
Pleural effusion $(\mathrm{n})$ & 0 & 0 & - \\
Incision infection $(\mathrm{n})$ & 1 & 0 & 0.30 \\
\hline
\end{tabular}

CAG CCC TCT G-3' and 5'-GAG GAC CTG GGA GTA GAT GAG-3'); IL-6 (5'-ACT CAC CTC TTC AGA ACG AAT TG-3' and 5'-CCA TCT TTG GAA GGT TCA GGT TG-3'); IL-10 (5'-GAC TTT AAG GGT TAC CTG GGT TG-3' and 5'-TCA CAT GCG CCT TGA TGT CTG-3'); and $\beta$-actin: 5'-AGC GAG CAT CCC CCA AAG TT-3' and 5'-GGG CAC GAA GGC TCA TCA TT-3'). $\beta$-actin was used as an endogenous control.

\section{Serum MDA and SOD assay}

MDA and SOD are commonly used as markers to estimate oxidative stress (19). Serum MDA was measured using kits and performed according to the manufacturer's directions (Jiangcheng Biotechnology, Nanjing, China). The results are expressed in nmol MDA per liter of serum. SOD activity was measured by an indirect competition assay between SOD and the indicator compound, nitroblue tetrazolium (NBT), for the superoxide produced by xanthine/xanthine oxidase according to the method of Spitz and Oberley. The data are expressed as $\mu \mathrm{mol} / \mathrm{L}$.

\section{Statistical analysis}

Data are presented as mean \pm SD. SPSS software (Chicago, IL, USA) was used to calculate the statistical significance by performing one-way analysis of variance. The $\chi^{2}$ test was used, when appropriate, for analysis of categorical data. All of the $\mathrm{P}$ values were two-sided, and $\mathrm{P}<0.05$ was considered to be statistically significant.

\section{Results}

\section{Demographic data from the two groups}

We randomized 80 patients, of whom 17 were later excluded; occlusion of hepatic blood flow or hepatic resection was not performed in 11 patients for poor liver reserve function (7 in the Fasting group and 4 in the Ctrl group), the hepatic artery was ligated in 4 patients ( 1 in the Fasting group and 3 in the Ctrl group), and another 2 patients did not continue to fast due to nurses misleading messages ( 2 in the Fasting group). The final number of patients eligible for analysis was 63 (30 in the Fasting group and 33 in the Ctrl group). No differences were observed in clinical and demographic data, including operative time, intraoperative blood loss, tumor types and sizes, time of clamping portal hepatis and the extent of liver resection BCLC stage, JIS score, platelets, prothrombin time, AFP, ICG-15, \% (Table 1). Complications occurring throughout the entire hospitalization did not differ between the groups. Details of postoperative complications are reported in Table 2.

\section{Short-term fasting improves hepatic function in patients undergoing hepatectomy}

We first determined the effects of short-term fasting on hepatic function in patients undergoing hepatectomy. Transaminase (ALT and AST), albumin, and total bilirubin (TBIL) were collected to assess the liver injury preoperation and at 1,3 , and 7 days post-operation. As shown in Figure 2A,B, sALT and sAST levels were significantly increased at POD-1, POD-3, and POD-7 compared with the pre-operation levels, especially at 1 - and 3-day postoperation $(\mathrm{P}<0.05)$. Interestingly, preoperative $24 \mathrm{~h}$ fasting effectively attenuated an increase of sALT and sAST after hepatectomy, especially at 1 - and 3-day post-operation $(\mathrm{P}<0.05)$. In addition, albumin and total bilirubin were analyzed, and there was no significant difference between the Ctrl group and the Fasting group (Figure 2C,D); but total bilirubin more rapidly declined in the Fasting group compared with the Ctrl group at 3 days post-operation $(\mathrm{P}<0.05$; Figure 2D). These data indicate that preoperative short-term fasting effectively attenuates surgery-related liver injury.

\section{Short-term fasting reduces inflammatory response in patients undergoing bepatectomy}

Next, we estimated the effects of short-term fasting on the inflammatory response in patients undergoing hepatectomy. Gene expression of pro-inflammatory cytokines (TNF- $\alpha$ and IL-6) and anti-inflammatory cytokines (IL-10) were examined in ischemic liver tissues. 
A

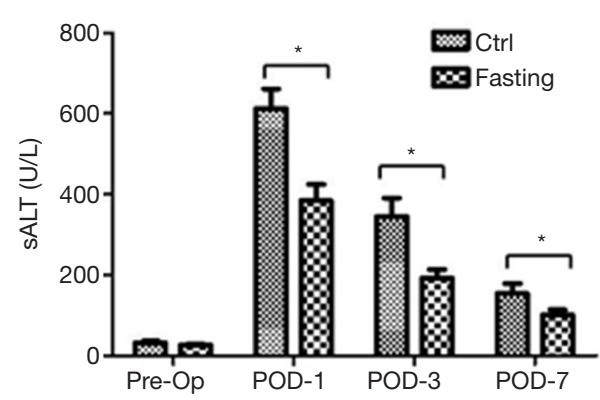

C

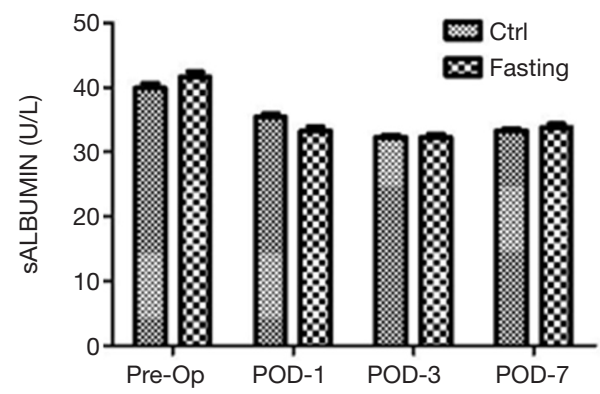

B

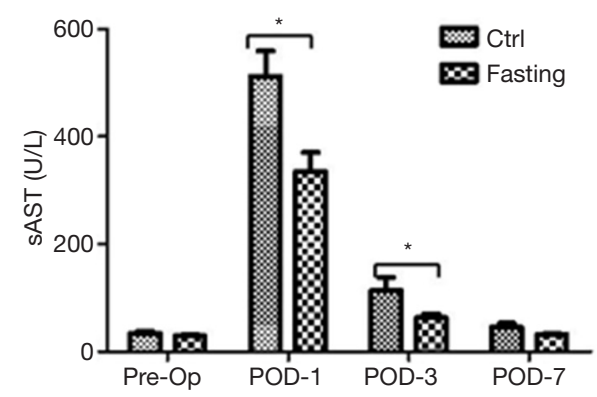

D

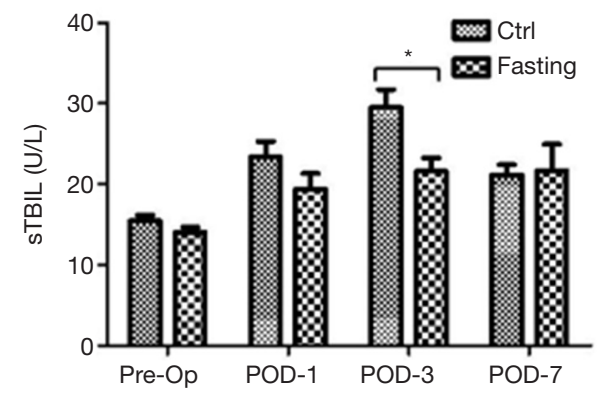

Figure 2 Twenty-four hours fasting improves hepatic function in patients undergoing hepatectomy. All patients were subjected to hepatectomy. Some patients were fasted $24 \mathrm{~h}$ prior to surgery. sALT (A), sAST (B), serum album (C), and total bilirubin (D) were collected to assess liver injury at pre-operation, and 1,3 and 7 days after operation. (mean $\pm \mathrm{SD},{ }^{*}, \mathrm{P}<0.05$ vs. Ctrl group).

Figure $3 A$ shows that fasting significantly inhibited the surgery-triggered expression of TNF- $\alpha(23.39 \pm 7.42$ and $2.96 \pm 0.78$, respectively; $\mathrm{P}<0.05$ ), but there was no difference in IL- 6 and IL-10 at the gene level between the Ctrl group and the Fasting group (Figure $3 A$ ). We further analyzed the expression of these cytokines in serum using ELISA and they were consistent with the gene expressions of the liver tissues. Figure $3 B$ showed that fasting markedly attenuated the surgery-triggered expression of TNF- $\alpha$ in serum compared with the $\mathrm{Ctrl}$ group $(\mathrm{P}<0.05)$; however, the expressions of IL-6 and IL-10 were not significantly different at the protein levels in serum of both of the groups (Figure 3C,D). Generally, the above data reflect that preoperative short-term fasting effectively attenuates the inflammatory response, especially TNF- $\alpha$ expression.

\section{Short-term fasting attenuates oxidative stress in patients undergoing bepatectomy}

Oxidative stress plays a prominently causative role in liver injury following hepatectomy (20). Oxidative stress can trigger the cascade of cell damage, necrosis/apoptosis, and subsequent pro-inflammatory responses. MDA, an index of lipid peroxidation that indicates the levels of the oxygen free radicals, is significantly increased after liver resection (21). MDA was significantly decreased in the Fasting group compared with the Ctrl group at POD-3 and POD-7, but not at POD-1 (Figure 4A). In addition, antioxidant SOD was measured in serum, which showed that SOD was significantly decreased after surgery. Fortunately, SOD activity gradually recovered as time goes by. Interestingly, pre-operation fasting accelerated the recovery process compared with the Ctrl group (Figure $4 B$ ). These data indicate pre-operation short-term fasting attenuates oxidative stress after hepatectomy.

\section{Short-term fasting activates nuclear factor erythroid- derived 2-related factor 2 (Nrf2) signaling pathway in liver tissue}

The Nrf2, a member of the Cap'n'Collar subfamily of bZIP transcription factor family, plays a pivotal role in the defense system against inflammatory responses and oxidative stress. To further investigate relative mechanisms of protective 

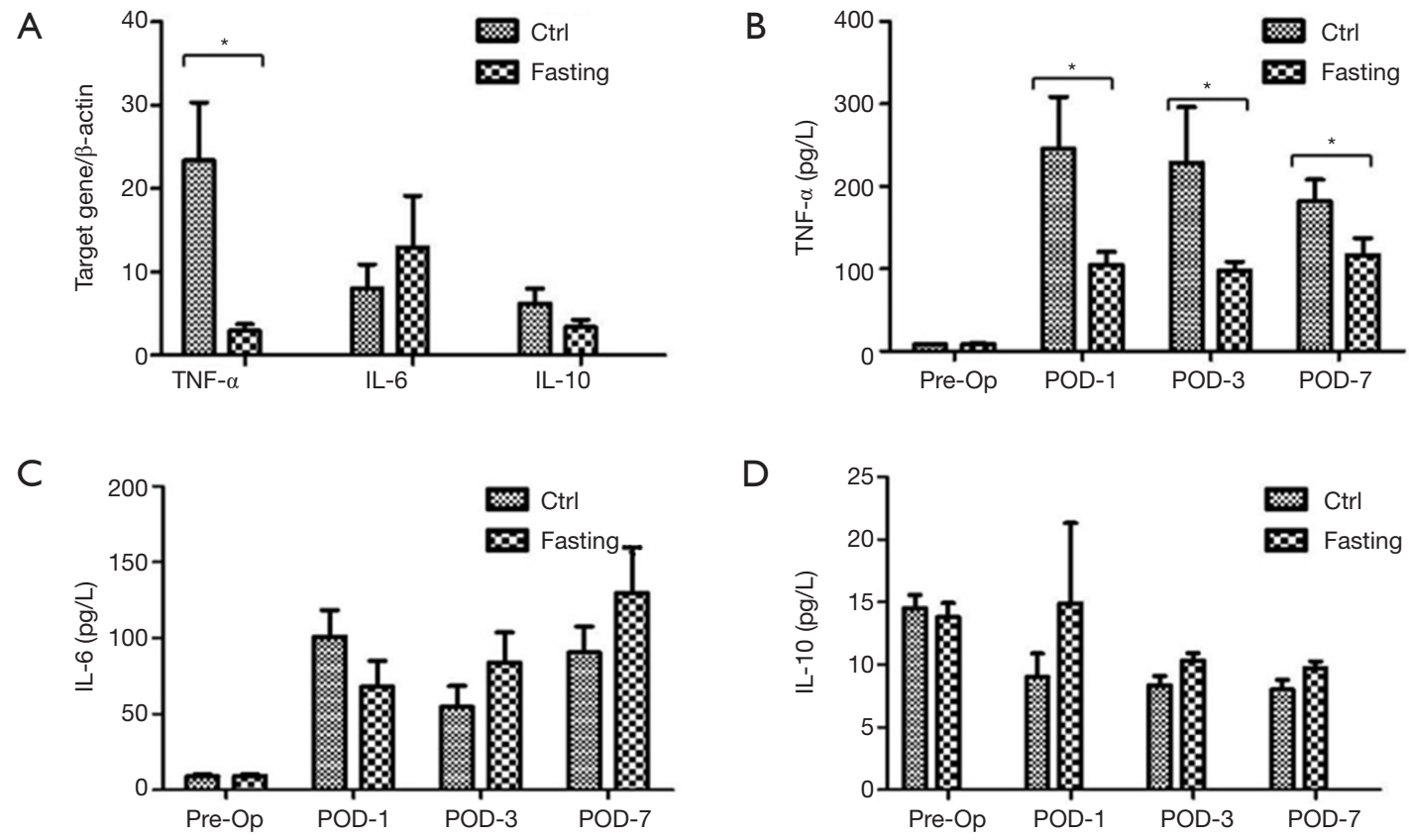

Figure 3 Twenty-four hours fasting reduces inflammatory response in patients undergoing hepatectomy. All patients were subjected to hepatectomy. Some patients were fasted $24 \mathrm{~h}$ prior to surgery. Expression of TNF- $\alpha$, IL-6 and IL-10 (A) was analyzed by real-time PCR in liver tissue. Serum TNF- $\alpha$ (B), IL-6 (C) and IL-10 (D) were measured to assess inflammatory responses by ELISA at pre-operation, and 1 day, 3 and 7 days after operation. (mean $\pm \mathrm{SD},{ }^{*}, \mathrm{P}<0.05$ vs. Ctrl group).
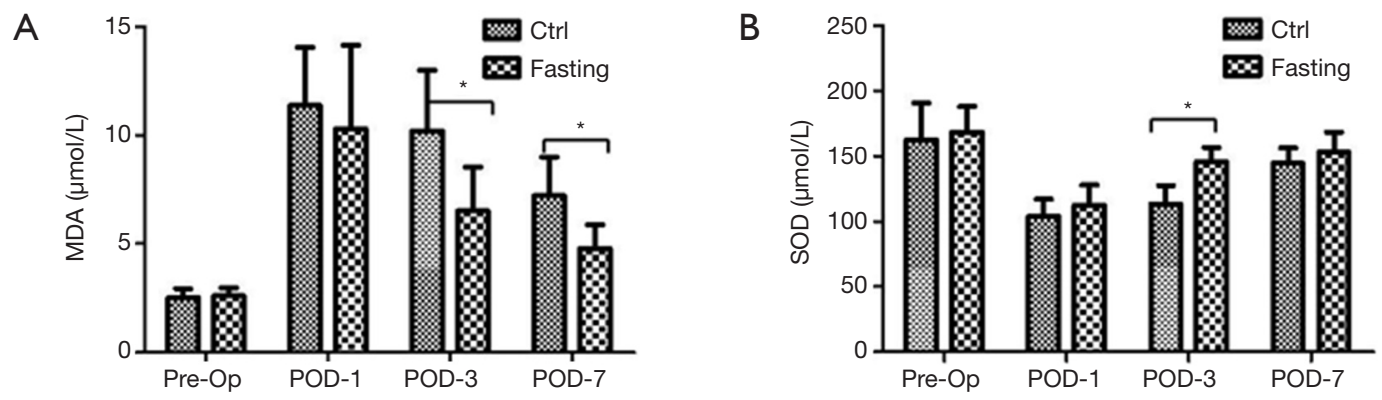

Figure 4 Twenty-four hours fasting attenuates oxidative stress in patients undergoing hepatectomy. All patients were subjected to hepatectomy. Some patients were fasted $24 \mathrm{~h}$ prior to surgery. Serum MDA (A) and SOD (B) were measured to assess oxidative stress by kits at pre-operation, and 1,3 and 7 days after operation. (mean $\pm \mathrm{SD},{ }^{*}, \mathrm{P}<0.05$ vs. Ctrl group).

effects of short-term fasting on hepatectomy, Nrf2 signaling pathway was analyzed in liver specimens. As shown in Figure $5 A$, Nrf2 mRNA expression was significantly increased in fasting group compared with ctrl group. In addition, Nrf2 related genes, HO-1 and Nqo1, were further analyzed, which showed mRNA expression of HO-1 and Nqo1 were also markedly increased in fasting group (Figure $5 A$ ). Next, Nrf2 protein expression further determined by Western blot, which supported above results (Figure 5B). These data indicate short-term fasting activates Nrf2 signaling pathway.

\section{Discussion}

Liver IRI remains the major cause of liver dysfunction and failure after hepatectomy and liver transplantation $(22,23)$. 
A

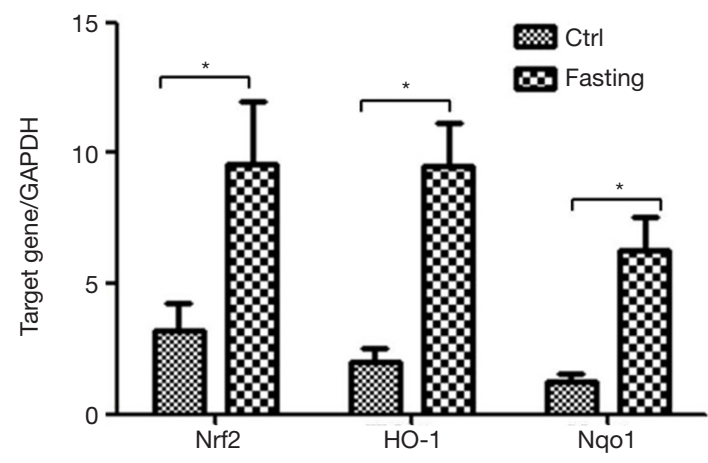

B

Nrf2

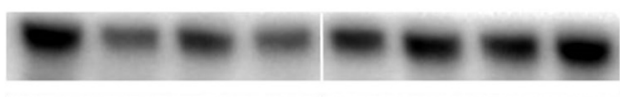

$\beta$-actin

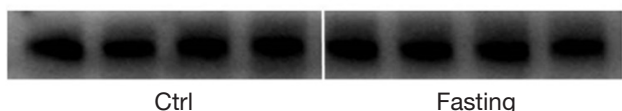

Figure 5 Twenty-four hours fasting activates Nrf2 signaling pathway in liver tissue. All patients were subjected to hepatectomy. Some patients were fasted $24 \mathrm{~h}$ prior to surgery. Expression of Nrf2, HO-1 and Nqo1 (A) was analyzed by real-time PCR in liver tissue; (B) expression of Nrf2 was analyzed by Western blot in liver tissue (mean $\pm \mathrm{SD}, *, \mathrm{P}<0.05$ vs. Ctrl group).

$\mathrm{CR}$ and fasting have displayed some beneficial effects on the prolongation of life and increased resistance to stress, including liver IRI. Our previous study demonstrated that short-term starvation, including starvation for 1, 2, and 3 days, attenuated liver IRI in the mouse model, which piqued our interest in its clinical application during liver resection (1). Herein, we designed a prospective, singleblinded, randomized study to analyze the effects of preoperative short-term fasting on hepatectomy. One day (24 h), as a period of short-term fasting, is an acceptable and feasible time span for patients. To our knowledge, this is the first study analyzing the effects of short-term fasting on hepatic IRI after hepatectomy in human.

First, we collected the pre- and intra-operative demographic data, including age, gender, tumor size, tumor type, ischemic time, operation time, blood loss, and so on (Table 1). After analyzing the data, we noted that there was not a marked difference between the two groups, so comparing post-operative outcomes from both groups was reasonable. Next, we estimated the effects of fasting on liver injury by biochemical outcomes, which showed that $24 \mathrm{~h}$ fasting effectively attenuated the increase of sALT and sAST after operation. In addition, $24 \mathrm{~h}$ fasting relatively stabilized the effects of surgery on serum TBIL, but fasting did not affect the level of serum albumin. Furthermore, postsurgical complications of both groups were similar during the hospitalization of the patients. Taken together, short-term fasting improves the clinical outcomes to some extent in patients undergoing liver resection. This finding is consistent with our previous research, in which shortterm starvation attenuated liver injury-induced by ischemia/ reperfusion in the mouse liver IRI model (1). Johnson's group also demonstrated that alternate-day CR improved symptoms and pulmonary function in overweight adults with moderate asthma (24). In addition, CR or fasting improves numerous health indicators, including those associated with risk of cardiovascular disease, type 2 diabetes and cancers, in rodents, monkeys, and humans (25-28).

The inflammatory response is an important risk factor of liver injury after hepatectomy, especially innate immune responses involved in inflammatory cytokines (29-31). In our study, pro- and anti-inflammatory cytokines, including TNF- $\alpha$, IL-6, and IL-10, were analyzed in liver tissues and serum. The reductions in the expression of TNF- $\alpha$ in liver tissues and serum of patients in the Fasting group compared to the Ctrl group were particularly striking; however, IL-6 and IL-10 did not significantly change in either of the groups. TNF- $\alpha$ is mainly responsible for the induction of neutrophil sequestration in the liver and directly mediates tissue injury (32-34), and TNF- $\alpha$ can activate hepatocellular programmed necrosis by the TNFR1 binding Fas-associated death domain (FADD) protein in many liver diseases (35). Thus, the decreased level of TNF- $\alpha$ suggests that $24 \mathrm{~h}$ fasting inhibits inflammatory responses and reduces hepatocellular death, which may contribute to the beneficial effects of fasting on clinical outcomes. In fact, Clavien's group and our group have demonstrated that one-day fasting downregulates the circulating levels of HMGB1, reduces inflammatory responses, and attenuates mouse hepatic IRI via the Sirt1autophagy pathway (36).

Oxidative stress is another risk factor of liver injury in many conditions, including liver IRI, hepatectomy, and so on (37-40). MDA, an end-product of lipid peroxidation, is an important criterion of oxidative stress and reflects the levels of tissue oxidative stress. In this study, levels of MDA were significantly lower in the Fasting group compared with the Ctrl group at POD-3 and POD-7, but not at POD-1, which indicated that $24 \mathrm{~h}$ fasting increased the scavenging ability of oxygen free radicals and accelerated the remission of oxidative stress. In addition, SOD, a classical antioxidant enzyme, increases susceptibility of the antioxidant system 
during liver IRI. In this study, $24 \mathrm{~h}$ fasting only increased the activity of SOD at POD-1 a little, but significantly enhanced the activity of SOD at POD-3, and it had almost the same level as the Ctrl group at POD-7. The above data indicated that fasting accelerated the recovery of SOD activity and then attenuated oxidative stress after hepatectomy, which was consistent with the MDA level. In parallel, fasting and postprandial blood glucose increments contributed to the oxidative stress and inflammation in dyslipidemic type 2 diabetic patients with stable ischemic heart disease (41).

Nrf2 plays a key role in the defense system against inflammatory responses and oxidative stress in various diseases conditions. Under homeostatic conditions, Nrf2 levels are maintained at low levels due to E3 ubiquitin ligase-mediated ubiquitylation and proteasome-dependent degradation of Nrf2 (42). Under stress conditions, Nrf2 degradation was markedly inhibited in the liver, heart, and brain (43-45). Activation of Nrf2 induces the expression of HO-1 and Nqo1, suggesting that Nrf2 is essential for the regulation of HO-1 and Nqo1. Numerous studies have indicated that Nrf2 signaling pathway plays a key role in inflammatory responses and oxidative stress during IRI $(46,47)$. Our data displayed $24 \mathrm{~h}$ fasting effectively activates Nrf2 signaling pathway in liver tissues. Thus, Nrf2 signaling pathway may play critical role in short-term fasting against inflammatory responses and oxidant stress.

In summary, our findings show (I) $24 \mathrm{~h}$ fasting effectively improves serum hepatic biochemistry in patients undergoing hepatectomy; and (II) the fasting-associated protection feature may be closed related to reduced inflammation and oxidative stress; (III) Nrf2 signaling pathway may play key role short-term fasting against inflammatory responses and oxidant stress. Preoperative short-term fasting is inexpensive, noninvasive, and very attractive to implement in the clinical setting; however, since we do not know the exact fasting duration, including 1, 2, or 3 days, to induce the greatest advantages in patients, further clinical trials will need to be performed.

\section{Acknowledgements}

We thank LetPub (www.letpub.com) for its linguistic assistance during the preparation of this manuscript.

Funding: This study was supported by the Foundation of Jiangsu Collaborative Innovation Center of Biomedical Functional Materials, the Priority Academic Program Development of Jiangsu Higher Education Institutions, the National Natural Science Foundation of China (81871259),
Six talent peaks project in Jiangsu Province (2017-WSW019), Huai'an science and technology planning project (HAB201740).

\section{Footnote}

Conflicts of Interest: The authors have no conflicts of interest to declare.

Ethical Statement: The Research Ethics Committee of the First Affiliated Hospital of Nanjing Medical University, in Nanjing, China (Ref: 2014/185) granted permission for the study protocol, and the trial was registered at the beginning of the study (Ref: ChiCTR-TRC-11001530, September 2014).

\section{References}

1. Qin J, Zhou J, Dai X, et al. Short-term starvation attenuates liver ischemia-reperfusion injury (IRI) by Sirt1-autophagy signaling in mice. Am J Transl Res 2016;8:3364-75.

2. Management of hepatocellular carcinoma. Clinical practice guidelines of European Association for the Study of the Liver (EASL), European Organisation for Research and Treatment of Cancer (EORTC). J Hepatol 2012;56:908-43.

3. Papadopoulos D, Siempis T, Theodorakou E, et al. Hepatic ischemia and reperfusion injury and trauma: current concepts. Arch Trauma Res 2013;2:63-70.

4. Scrofano MM, Shang F, Nowell TR Jr, et al. Calorie restriction, stress and the ubiquitin-dependent pathway in mouse livers. Mech Ageing Dev 1998;105:273-90.

5. McCay CM, Crowell MF, Maynard LA. The effect of retarded growth upon the length of the life span and upon the ultimate body size. J Nutr 1935;10:63-79.

6. Chandrasekar B, Nelson JF, Colston JT, et al. Calorie restriction attenuates inflammatory responses to myocardial ischemia-reperfusion injury. Am J Physiol Heart Circ Physiol 2001;280:H2094-102.

7. Mitchell JR, Beckman JA, Nguyen LL, et al. Reducing elective vascular surgery perioperative risk with brief preoperative dietary restriction. Surgery 2013;153:594-8.

8. Weindruch R, Walford RL. Dietary restriction in mice beginning at 1 year of age: effect on life-span and spontaneous cancer incidence. Science 1982;215:1415-8.

9. Sohal RS, Weindruch R. Oxidative stress, caloric restriction, and aging. Science 1996;273:59-63.

10. Ramsey JJ, Harper ME, Weindruch R. Restriction of energy intake, energy expenditure, and aging. Free Radic Biol Med 
2000;29:946-68.

11. Goodrick CL, Ingram DK, Reynolds MA, et al. Effects of intermittent feeding upon growth, activity, and lifespan in rats allowed voluntary exercise. Exp Aging Res 1983;9:203-9.

12. Mitchell JR, Verweij M, Brand K, et al. Short-term dietary restriction and fasting precondition against ischemia reperfusion injury in mice. Aging Cell 2010;9:40-53.

13. Shirodkar JA, Sayyad MG, Nanal VM, et al. Anguli Parimana in Ayurveda and its association with adiposity and diabetes. J Ayurveda Integr Med 2014;5:177-84.

14. Beshyah S, Benbarka M, Sherif I. Practical management of diabetes during Ramadan fast. Libyan J Med 2007;2:185-9.

15. Weiss J. Fasting in Ramadan despite chronic diseases. Dtsch Med Wochenschr 2009;134:29-30.

16. Hassanein M, Abdallah K, Schweizer A. A doubleblind, randomized trial, including frequent patientphysician contacts and Ramadan focused advice, assessing vildagliptin and gliclazide in patients with type 2 diabetes fasting during Ramadan: the STEADFAST study. Vasc Health Risk Manag 2014;10:319-26.

17. Bengmark S, Rosengren K. Angiographic study of the collateral circulation to the liver after ligation of the hepatic artery in man. Am J Surg 1970;119:620-4.

18. Olthof PB, van Golen RF, Meijer B, et al. Warm ischemia time-dependent variation in liver damage, inflammation, and function in hepatic ischemia/reperfusion injury. Biochim Biophys Acta Mol Basis Dis 2017;1863:375-85.

19. Mateos R, Lecumberri E, Ramos S, et al. Application to a rat model for hypercholesterolemia and evaluation of the effect of diets rich in phenolic antioxidants from fruits. J Chromatogr B Analyt Technol Biomed Life Sci 2005;827:76-82.

20. Hori T, Uemoto S, Chen F, et al. Oxidative stress and extracellular matrices after hepatectomy and liver transplantation in rats. World J Hepatol 2014;6:72-84.

21. Hassan HM, Guo H, Yousef BA, et al. Role of Inflammatory and Oxidative Stress, Cytochrome P450 2E1, and Bile Acid Disturbance in Rat Liver Injury Induced by Isoniazid and Lipopolysaccharide Cotreatment. Antimicrob Agents Chemother 2016;60:5285-93.

22. Henderson JM. Liver transplantation and rejection: an overview. Hepatogastroenterology 1999; 46:1482-4.

23. Howard TK, Klintmalm GB, Cofer JB, et al. The influence of preservation injury on rejection in the hepatic transplant recipient. Transplantation 1990;49:103-7.

24. Johnson JB, Summer W, Cutler RG, et al. Alternate day calorie restriction improves clinical findings and reduces markers of oxidative stress and inflammation in overweight adults with moderate asthma. Free Radic Biol Med 2007;42:665-74.

25. Most J, Tosti V, Redman LM, et al. Calorie restriction in humans: An update. Ageing Res Rev 2017;39:36-45.

26. Carter S, Clifton PM, Keogh JB. Intermittent energy restriction in type 2 diabetes: A short discussion of medication management. World J Diabetes 2016;7:627-30.

27. Shang Y, Kakinuma S, Yamauchi K, et al. Cancer prevention by adult-onset calorie restriction after infant exposure to ionizing radiation in $\mathrm{B} 6 \mathrm{C} 3 \mathrm{~F} 1$ male mice. Int $\mathrm{J}$ Cancer 2014;135:1038-47.

28. Mirsoian A, Bouchlaka MN, Sckisel GD, et al. Adiposity induces lethal cytokine storm after systemic administration of stimulatory immunotherapy regimens in aged mice. J Exp Med 2014;211:2373-83.

29. Rao J, Qian X, Li G, et al. ATF3-mediated NRF2/HO-1 signaling regulates TLR4 innate immune responses in mouse liver ischemia/reperfusion injury. Am J Transplant 2015;15:76-87.

30. Rao J, Yue S, Fu Y, et al. ATF6 mediates a proinflammatory synergy between ER stress and TLR activation in the pathogenesis of liver ischemia-reperfusion injury. Am J Transplant 2014;14:1552-61.

31. Yue S, Rao J, Zhu J, et al. Myeloid PTEN deficiency protects livers from ischemia reperfusion injury by facilitating M2 macrophage differentiation. J Immunol 2014;192:5343-53.

32. Chang L, Kamata H, Solinas G, et al. The E3 ubiquitin ligase itch couples JNK activation to TNFalphainduced cell death by inducing c-FLIP(L) turnover. Cell 2006;124:601-13.

33. Descamps D, Vigant F, Esselin S, et al. Expression of nonsignaling membrane-anchored death receptors protects murine livers in different models of hepatitis. Hepatology 2006;44:399-409.

34. Brenner C, Galluzzi L, Kepp O, et al. Decoding cell death signals in liver inflammation. J Hepatol 2013;59:583-94.

35. Boutaffala L, Bertrand MJ, Remouchamps C, et al. NIK promotes tissue destruction independently of the alternative NF- $\kappa$ B pathway through TNFR1/RIP1induced apoptosis. Cell Death Differ 2015;22:2020-33.

36. Rickenbacher A, Jang JH, Limani P, et al. Fasting protects liver from ischemic injury through Sirt1-mediated downregulation of circulating HMGB1 in mice. J Hepatol 2014;61:301-8.

37. Tao X, Wan X, Xu Y, et al. Dioscin attenuates hepatic ischemia-reperfusion injury in rats through inhibition of 
Page 10 of 10

oxidative-nitrative stress, inflammation and apoptosis. Transplantation 2014;98:604-11.

38. Rao J, Zhang C, Wang P, et al. All-trans retinoic acid alleviates hepatic ischemia/reperfusion injury by enhancing manganese superoxide dismutase in rats. Biol Pharm Bull 2010;33:869-75.

39. Liu Y, Yang L, Tao K, et al. Protective effects of hydrogen enriched saline on liver ischemia reperfusion injury by reducing oxidative stress and HMGB1 release. BMC Gastroenterol 2014;14:12.

40. Rao J, Zhang C, Wang P, et al. C/EBP homologous protein (CHOP) contributes to hepatocyte death via the promotion of ERO $1 \alpha$ signalling in acute liver failure. Biochem J 2015;466:369-78.

41. Djindjic B, Kostic T, Radovanovic Z, et al. The contributions of fasting and postprandial blood glucose increments to oxidative stress and inflammation in dyslipidemic type 2 diabetic patients with stable ischemic heart disease. Int J Cardiol 2017;227:611-6.

42. Kobayashi A, Kang MI, Okawa H, et al. Oxidative stress sensorKeap1 functions as an adaptor for Cul3-based E3

Cite this article as: Zhan C, Dai X, Shen G, Lu X, Wang X, Lu L, Qian X, Rao J. Preoperative short-term fasting protects liver injury in patients undergoing hepatectomy. Ann Transl Med 2018;6(23):449. doi: 10.21037/atm.2018.10.64
Zhan et al. Short-term fasting in patients undergoing hepatectomy

ligase to regulate proteasomal degradation of $\mathrm{Nrf} 2 . \mathrm{Mol}$ Cell Biol 2004;24:7130-9.

43. Ke B, Shen XD, Zhang Y, et al. KEAP1-NRF2 complex in ischemia induced hepatocellular damage of mouse liver transplants. J Hepatol 2013;59:1200-7.

44. Deng C, Sun Z, Tong G, et al. a-Lipoic acid reduces infarct size and preserves cardiac function in rat myocardial ischemia/reperfusion injury through activation of PI3K/ Akt/Nrf2 pathway. PLoS One 2013;8:e58371.

45. Porritt MJ, Andersson HC, Hou L, et al. Photothrombosis-induced infarction of the mouse cerebral cortex is not affected by the Nrf2-activator sulforaphane. PLoS One 2012;7:e41090.

46. Ke B, Shen XD, Ji H, et al. HO-1-STAT3 axis in mouse liver ischemia/reperfusion injury: Regulation of TLR4 innate responses through PI3K/PTEN signaling. J Hepatol 2012;56:359-66.

47. Shen XD, Ke B, Ji H, et al. Disruption of type-I IFN pathway ameliorates preservation damage in mouse orthotopic liver transplantation via HO-1 dependent mechanism. Am J Transplant 2012;12:1730-9. 